\title{
Characterization of an ester-based core-multishell (CMS) nanocarrier for the topical application at the oral mucosa
}

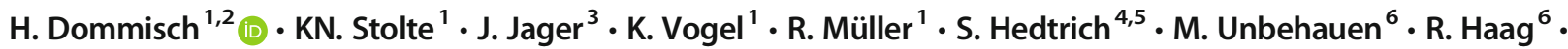 \\ K. Danker ${ }^{3}$
}

Received: 28 September 2020 / Accepted: 11 March 2021 / Published online: 5 April 2021

(C) The Author(s) 2021

\begin{abstract}
Objectives Topical drug administration is commonly applied to control oral inflammation. However, it requires sufficient drug adherence and a high degree of bioavailability. Here, we tested the hypothesis whether an ester-based core-multishell (CMS) nanocarrier is a suitable nontoxic drug-delivery system that penetrates efficiently to oral mucosal tissues, and thereby, increase the bioavailability of topically applied drugs.

Material and methods To evaluate adhesion and penetration, the fluorescence-labeled CMS 10-E-15-350 nanocarrier was applied to ex vivo porcine masticatory and lining mucosa in a Franz cell diffusion assay and to an in vitro 3D model. In gingival epithelial cells, potential cytotoxicity and proliferative effects of the nanocarrier were determined by MTT and sulphorhodamine $\mathrm{B}$ assays, respectively. Transepithelial electrical resistance (TEER) was measured in presence and absence of CMS 10-E-15-350 using an Endohm-12 chamber and a volt-ohm-meter. Cellular nanocarrier uptake was analyzed by laser scanning microscopy. Inflammatory responses were determined by monitoring pro-inflammatory cytokines using real-time PCR and ELISA.

Results CMS nanocarrier adhered to mucosal tissues within $5 \mathrm{~min}$ in an in vitro model and in ex vivo porcine tissues. The CMS nanocarrier exhibited no cytotoxic effects and induced no inflammatory responses. Furthermore, the physical barrier expressed by the TEER remained unaffected by the nanocarrier.

Conclusions CMS 10-E-15-350 adhered to the oral mucosa and adhesion increased over time which is a prerequisite for an efficient drug release. Since TEER is unaffected, CMS nanocarrier may enter the oral mucosa transcellularly.

Clinical relevance Nanocarrier technology is a novel and innovative approach for efficient topical drug delivery at the oral mucosa.
\end{abstract}

Keywords Core-multishell nanocarrier · Transepithelial resistance $\cdot$ Penetration $\cdot$ Oral mucosal equivalents

H. Dommisch and KN. Stolte contributed equally to this work.

H. Dommisch

henrik.dommisch@charite.de

1 Department of Periodontology, Oral Medicine and Oral Surgery, Charité - Universitätsmedizin Berlin, corporate member of Freie Universität Berlin, Humboldt-Universität zu Berlin, and Berlin Institute of Health, 14197 Berlin, Germany

2 Department of Periodontology, University of Washington, Seattle, WA, USA
3 Institute for Biochemistry, Charité - Universitätsmedizin Berlin, corporate member of Freie Universität Berlin, Humboldt-Universität zu Berlin, and Berlin Institute of Health, 10117 Berlin, Germany

4 Pharmacology and Toxicology, Institute of Pharmacy, Freie Universität Berlin, 14195 Berlin, Germany

5 Faculty of Pharmaceutical Sciences, University of British Columbia, 2405 Wesbrook Mall, Vancouver, BC V6T1Z3, Canada

6 Institute for Chemistry and Biochemistry, Freie Universität Berlin, 14195 Berlin, Germany 


\section{Introduction}

In the oral cavity, the epithelial surface is constantly exposed to high numbers of not only highly variable microorganisms, but also chemical, thermal, and mechanical environmental factors. The epithelial tissues exhibit effective physical as well as chemical defense properties that prevent infection and physical tissue damage. In some cases, however, this robust epithelial barrier may be more vulnerable and develop various inflammatory disease characteristics.

Periodontitis is a highly prevalent bacterially induced inflammatory disease along with epithelial barrier and bone tissue breakdown [1-3]. Besides mechanical treatment strategies, systemic antibiotics have been implemented to control the subgingival microbial flora. The adjunctive application of systemic antibiotics led to the reduction of periodontal pockets and inflammation $[4,5]$. However, only a few patients with severe periodontitis benefit from intake of adjunctive systemic antibiotics when disease progression was analyzed, and the overall clinical effect was rather marginal [6]. Furthermore, the resistance of subgingival microflora against antibiotics is increased, if systemic antibiotics are frequently administered in a population $[7,8]$. In addition, drug-mediated anti-inflammatory periodontal therapy is under current scientific discussion, and on the experimental level, promising approaches have been described $[9,10]$.

In addition to periodontitis, numerous other inflammatory conditions may, even simultaneously, be present at mucosal sites in the oral cavity. Lichen planus, Pemphigus vulgaris, and bullous mucosal pemphigoid not only are auto-immune diseases that are not restricted to the skin, but also show their characteristics on the oral mucosa [11]. Here, administration of topical drug formulations (e.g., cortisone) in form of cremes and mouth rinses is considered the standard therapeutical approach [12].

Topical application of drugs offers non-invasive feasibility, less side effects compared to oral or intravenous application, improved patient compliance, and increased bioavailability by avoiding the hepatic first-pass effect. However, topical application of any antiseptic and/or anti-inflammatory formulation is of reduced effectiveness for the patient due to limited bioavailability of current topical treatment approaches, time of application, and modes of application [13]. In general, many drugs show weak adherence to oral mucosal surfaces as well as in periodontal mucosal pockets due the constant flow of saliva (on average, 500-600 ml/day), mechanical drug displacement during chewing, and constant exudation of gingival crevicular fluid, a serum-like exudate secreted into the periodontal space [14-16].

Therefore, there is a strong medical need for novel therapeutic strategies for the treatment of periodontitis and other inflammatory diseases of the oral mucosa that combine a high grade of drug bioavailability and a safe regimen. Nanocarrier technology has the potential to overcome issues regarding bioavailability of any drug applied at mucosal sites. Even the special inflammatory environment may be utilized to increase the efficiency of application using photo-triggered, $\mathrm{pH}$ triggered, protease-triggered, or temperature-triggered release mechanisms [17-22].

Recently, we introduced the concept of core-multishell (CMS) nanocarrier as a novel carrier system to increase bioavailability of topically applied dexamethasone at the oral mucosa [23] (Fig. 1). The hPG-amide-C18-PEG-coremultishell nanocarrier (CMS 10-A-18-350) was applied to different porcine mucosal surfaces and showed excellent penetration properties [23]. In an ex vivo approach, we were able to show that dexamethasone loaded to a CMS nanocarrier was more efficiently released and taken up by oral mucosal tissues compared to dexamethasone from a conventional cream formulation [23]. However, it has been shown that application of the CMS 10-A-18-350 nanocarrier exhibited cytotoxic effects at high concentrations and after longer exposure times [23].

In the present study we, therefore, analyzed a novel generation of biodegradable, ester-based CMS nanocarriers. One of these newly developed nanocarriers, the CMS 10-E-15-350, was identified as the most biocompatible carrier in different models [25]. Inskin, the CMS 10-E-15-350 was identified as the most promising carrier [25]. Therefore, we chose and evaluated this specific nanocarrier for its ability to adhere and penetrate into oral mucosal tissues.

We hypothesized that the ester-based CMS 10-E-15-350 shows efficient mucosal penetration properties without inflammatory, cytotoxic or proliferative side effects. We found that penetration of CMS 10-E-15-350 can be observed within minutes after mucosal application. We did not detect any influence on proliferation and the metabolic activity. Application of the nanocarrier did not provoke an inflammatory response of gingival epithelial cells and the physical barrier was not altered since the transepithelial electrical resistance remained unaffected. Therefore, we expect excellent biocompatibility of this nanocarrier generation in future studies.

\section{Material and methods}

\section{Core-multishell nanocarrier 10-E-15-350}

The synthesis of the CMS 10-E-15-350 nanocarrier was recently described in [25]. The nanocarrier was soluble in culture medium. Dynamic light scatting measurements revealed that $71 \%$ of the nanocarrier particles solution had a size of $22.5 \mathrm{~nm}$.

For penetration and uptake experiments, the respective fluorescence-labeled version of the CMS ester-based nanocarrier (CMS 10-cICC-E-15-350, DendroPharm, Berlin, Germany) was used at a concentration of $10 \mathrm{mg} / \mathrm{ml}$. As a 
Fig. 1 Schematic representation of the dendritic core-multishell nanocarrier [24]. This type of nanocarrier allows loading of hydrophilic as well as of hydrophobic drugs and topical delivery at sites of inflammation

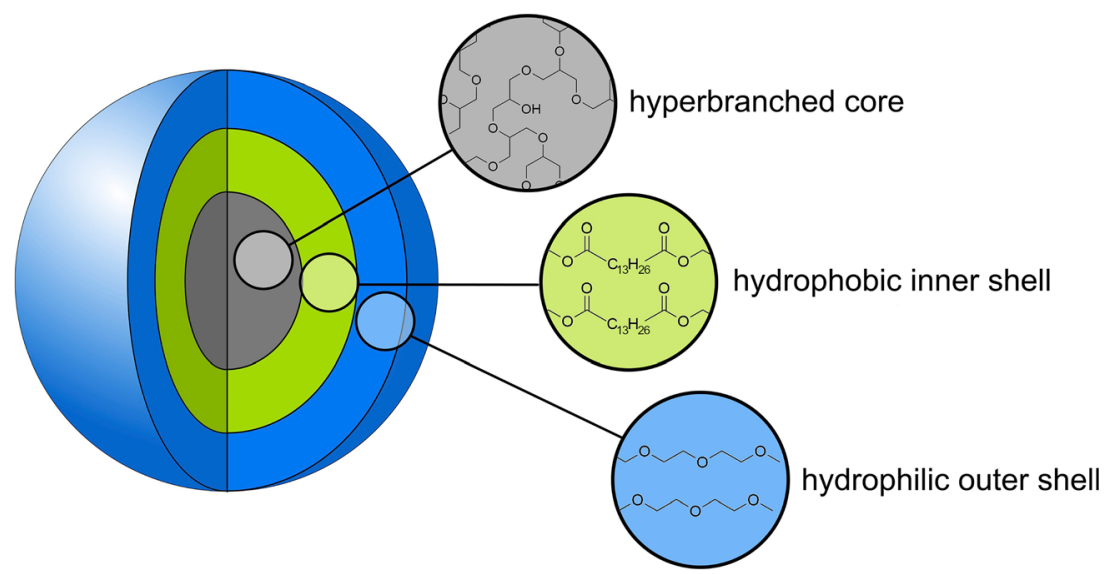

fluorescence label, the dye indocarbocyanine (ICC) was utilized. For all other experiments, the non-labeled carrier was used (Fig. 1).

\section{Tissue and cell culture}

For ex vivo experiments, the untreated native mucosa was dissected from areas of both masticatory and lining mucosa obtained from pigs slaughtered for food industry reasons. Tissues were then immediately stored in PBS for transportation and subjected to Franz cell experiments as recently described [23]. For experiments with cell monolayers, the immortalized human gingival keratinocytes OKG4/bmil/TERT (OKG4; kindly provided by Susan Gibbs, Amsterdam) were used $[26,27]$. Primary gingival epithelial cells (GECs) were dissected from gingival tissue samples obtained from tooth extractions, and tissues were and kindly provided by the Department of Oral and Maxillofacial Surgery at Campus Virchow, Charité.

Keratinocytes were cultured in DermaLife K medium with $1 \%$ penicillin/streptomycin in the presence of $60 \mu \mathrm{M}$ or $1.4 \mathrm{mM} \mathrm{Ca}^{2+}$ as indicated (CellSystems, Troisdorf, Germany). Cells were cultivated in collagen-coated plates and flasks at $37^{\circ} \mathrm{C}$ and $5 \% \mathrm{CO}_{2}$.

\section{Franz cell experiments}

Penetration studies were performed as recently described [23]. Porcine oral mucosal tissues $(2 \mathrm{~cm}$ diameter) were mounted onto static-type Franz cells (diameter $7 \mathrm{~mm}$, volume $5 \mathrm{ml}$, PermeGear, Bethlehem, PA, USA), and the nanocarrier was applied onto the mucosal surface for the time points indicated. Microsections of PFA-embedded tissues were analyzed for nanocarrier adhesion and penetration using the confocal laser scanning microscope LSM700MAT (CLSM, Zeiss). Three independent experiments were performed using three biological replicates for both masticatory and lining mucosa at each time point. More detailed information is given in the Supplementary information.

\section{D culture of in vitro organotypic mucosal equivalents}

To establish a model for tracking nanocarrier penetration within the oral mucosal tissue, a 3D organotypic cell culture was established [28]. Here, OKG4 cells and the fibroblast cell line (T0026) were co-cultured.

Briefly, $1 \mathrm{ml}$ of a collagen I solution mixed with $4 \times 10^{5}$ cells/ml gingival fibroblasts was transferred on an acellular collagen sheet from bovine type I collagen $(0.77 \mathrm{mg} / \mathrm{ml}$; Nutragen ${ }^{\circledR}$, Advanced BioMatrix) in a Millicell ${ }^{\circledR}$ culture plate insert (30 mm diameter, pore size $0.4 \mu \mathrm{m}$; Merck). Inserts were placed in 6 -well plates and incubated at $37^{\circ} \mathrm{C}$ and $5 \%$ $\mathrm{CO}_{2}$ for $1 \mathrm{~h}$. Prior to the addition of OKG4 cells $\left(1 \times 10^{6}\right.$, DermaLife $\mathrm{K}$ medium, $60 \mu \mathrm{M} \mathrm{Ca}{ }^{2+}$, Lifeline Cell Technology), fibroblast-populated collagen gels were cultured for 5 days in DMEM (Corning) containing 10\% FCS (PANBiotech). For more detailed information, please see the Supplementary information.

\section{Cell viability assays}

Assays for the determination of cell viability were recently described by our group [23]. Briefly, MTT and sulphorhodamine B (SRB) assays were performed upon nanocarrier exposure to OKG4 cells. Cell viability was monitored for 24, 48, and $72 \mathrm{~h}$. A total of three experiments were performed with each consisted of six technical replications. For further analysis, mean values of untreated control cells were set at $100 \%$ in each of the three experiments. Test groups were normalized to untreated control cells. Graphs represent the three experimental sets, and error bars indicate standard deviations in the test groups when normalized to controls. Values $>80 \%$ predicted no cytotoxic effects. More detailed 
information is given in the supplementary electronic file (Supplementary information Figure 1 A,B).

\section{Measurement of the transepithelial electrical resistance}

For the measurement of the transepithelial electrical resistance (TEER), $12 \mathrm{~mm}$ Transwell ${ }^{\circledR}$ inserts with $0.4 \mu \mathrm{m}$ pore polycarbonate membranes (Corning) coated with collagen IV $(20 \mu \mathrm{g} / \mathrm{ml}$, Sigma-Aldrich) were used. OKG4 cells $\left(2.7 \times 10^{4}\right)$ in $500 \mu$ in DermaLife K medium containing $60 \mu \mathrm{M} \mathrm{Ca}^{2+}$ were seeded to the filter, and the medium was changed every 1-3 days. When cells reached confluence (approx. after 7 days), they were cultured in a medium containing $1.4 \mathrm{mM} \mathrm{Ca}^{2+}$ to induce terminal cell differentiation [29] and the formation of tight junctions [30]. Subsequently, $50 \mu \mathrm{g} / \mathrm{ml}$ of the nanocarrier solution was applied to the cells. As a control, the TEER of cells that were kept in DermaLife $\mathrm{K}$ medium containing $60 \mu \mathrm{M} \mathrm{Ca}^{2+}$ was determined. The TEER values were measured using an Endohm-12 chamber (World Precision Instruments) and a volt-ohm-meter (Millipore). After subtracting the blank filter's TEER, the value was multiplied by the filter area $\left(1.12 \mathrm{~cm}^{2}\right)$. Four independent experiments were performed in duplicate.

\section{Cellular uptake}

The $5 \times 10^{4}$ OKG4 cells/well in DermaLife medium containing $60 \mu \mathrm{M} \mathrm{Ca}^{2+}$ were seeded to 8-well Permanox slides (Nunc) and cultured for $24 \mathrm{~h}$. The cells were subsequently cultivated in $60 \mu \mathrm{M}$ or $1.4 \mathrm{mM} \mathrm{Ca}^{2+}$ for further $24 \mathrm{~h}$ and treated with the respective cell culture medium containing ICC-coupled CMS 10-E-15-350 at a final ICC concentration of $2 \mu \mathrm{g} / \mathrm{ml}$ for further $24 \mathrm{~h}$. The cell nuclei were stained with Hoechst 33342 dye (blue, Invitrogen) and cell membranes were stained with Wheat Germ Agglutinin (WGA) Alexa Fluor 488-conjugated (Invitrogen), which binds to Nacetylglucosamine and $\mathrm{N}$-acetylneuraminic acid moieties on the cell surface, for $30 \mathrm{~min}$. Subsequently, the cells were rinsed with PBS to remove the excess of dyes. The internalization was observed using the LSM700MAT (Zeiss) and analyzed by the ZEN software (Zeiss). Each analysis was performed in triplicate.

\section{qPCR and ELISA experiments}

Detailed information on the methodology of qPCR and ELISA experiments is displayed in the Supplementary information.

\section{Statistical analysis}

For statistical analyses (Graph Pad Prism; $P<0.05$ ), absolute values were calculated using one-way ANOVA corrected by the Dunnett's multiple comparison test and the Tukey's multiple comparison test. For SRB and MTT assays, mean values determined from control cells were set at $100 \%$ as reference for test groups. The figures display relative values given in \% (bars) with standard deviation (errors bars), and analysis was performed using the Holm-Sidak's multiple comparison test. Real-time PCR experiments were analyzed using the one-way ANOVA with correction for multiple testing (Dunnett's multiple comparison test). For mRNA expression analysis, only relative fold changes greater than 2fold were considered biologically relevant (marked by a dashed line). A $P$-value of $P<0.05$ was considered significant.

\section{Results}

\section{Time-dependent penetration and adherence of CMS nanocarriers in porcine mucosal tissues}

Penetration of the CMS 10-E-15-350 nanocarrier coupled to a fluorescent dye was analyzed using porcine masticatory and lining mucosa and confocal microscopy (Fig. 2a). After $6 \mathrm{~h}$, the nanocarrier penetrated into both mucosal tissue types (Fig. 2a). While the CMS nanocarrier penetrated evenly into the para-keratinized lining mucosa, it was prone to aggregation in the stratum corneum of the masticatory mucosa.

For clinical translation, shorter application times were tested. After $5 \mathrm{~min}$, the nanocarrier showed a sparse penetration pattern in the superior cell layer of the lining mucosa, whereas at the masticatory mucosa, the nanocarrier exhibited firm adhesion with no evidence for penetration. After $30 \mathrm{~min}$, the CMS nanocarrier penetrated into the lining mucosa with a comparable depth observed for the 6-h time point, while the majority of the nanocarrier only adhered to the stratum corneum of the masticatory mucosa (Fig. 2b).

\section{D culture technique as a novel experimental design to track nanocarrier penetration}

Additionally, we tested the penetration of the CMS 10-E-15350-ICC nanocarrier in an organotypic 3D model (Fig. 3). The multi-layered epidermal component was anchored to the underlying collagen/fibroblast gel (Fig. 3a). The epidermal cells expressed keratins (green) and the fibroblasts were positive for vimentin (red; Fig. 3b). The epidermal component 
Fig. 2 Representative images of nanocarrier penetration into ex vivo masticatory and lining porcine mucosa at various time points $(\mathbf{a}, \mathbf{b})$. Microsections were stained for pan-cytokeratin shown in green and cell nuclei visualized with the Hoechst 33342 dye (blue). The CMS 10-E-15-350 nanocarrier was labeled with the fluorescent dye indocarbocyanine (red). Magnification $\times 20$ a masticatory

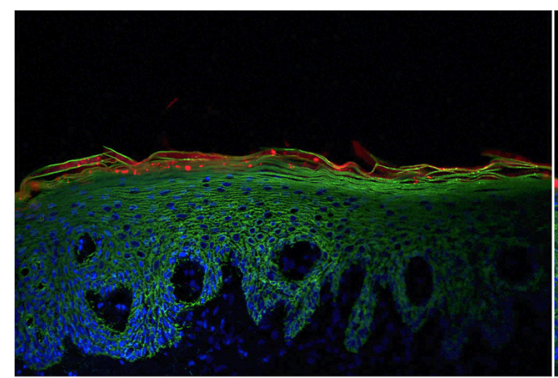

b
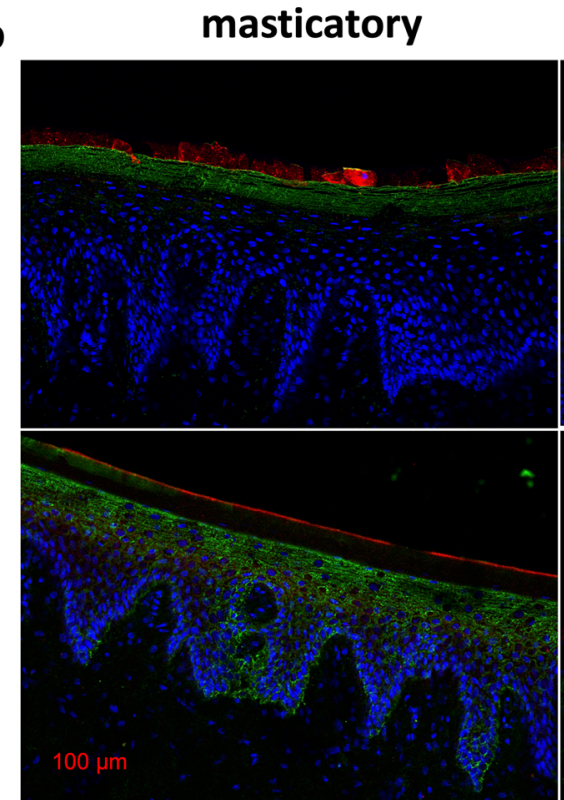

lining

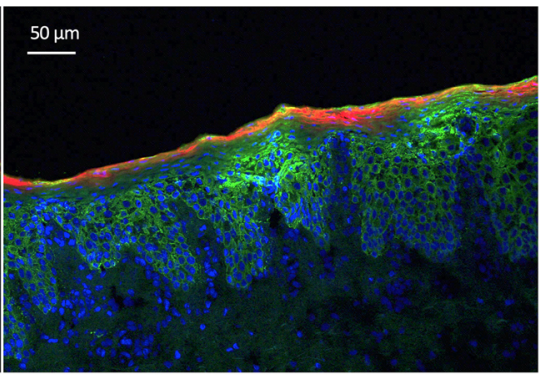

$6 \mathrm{~h}$

$5 \mathrm{~min}$

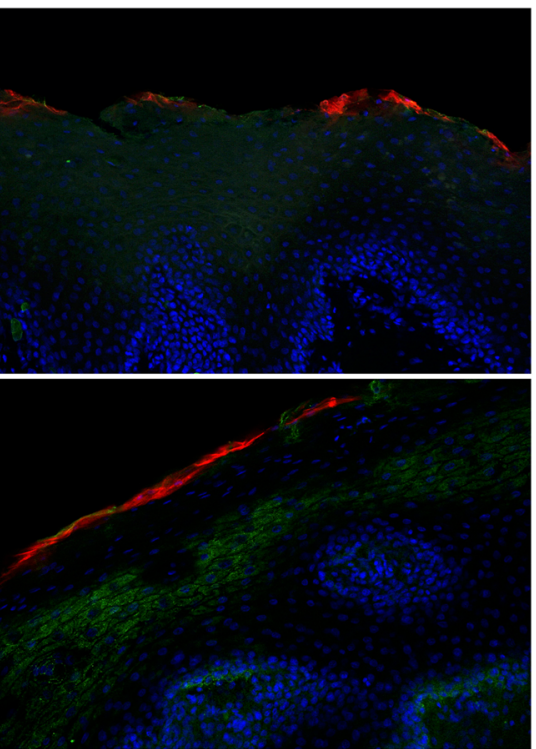

$30 \mathrm{~min}$ revealed clear signs of terminal differentiation since cells of the upper part expressed the differentiation marker filaggrin (Fig. 3c). Cells of the basal layer showed round cell bodies and nuclei, while epithelial cells from the upper layer changed their morphology to a plainer shape of cells and nuclei (Fig. $3 \mathrm{a}$ and $b$ ).

Nanocarrier application to the surface of the 3D model exhibited penetration into the epidermal compartment after 5 min. The collagen matrix underneath the epithelial cell layer was not penetrated by the nanocarrier (red, Fig. 3d).

\section{CMS 10-E-15-350 nanocarrier did not cause cytotoxic effects in OKG4 cells}

For each time point tested, cell proliferation of immortalized gingival keratinocytes was not affected by the nanocarrier compared to untreated control cells (Fig. 4a).

After $24 \mathrm{~h}$, the metabolic activity was increased by a nanocarrier concentrations of $50 \mu \mathrm{g} / \mathrm{ml}, 100 \mu \mathrm{g} / \mathrm{ml}$, and 500 $\mu \mathrm{g} / \mathrm{m}$. After 48 and $72 \mathrm{~h}$, an influence on the metabolic activity of cells could not be observed at any concentration tested (Fig. 4b). In primary gingival keratinocytes, an influence on the metabolic activity in the presence of 50 and $500 \mu \mathrm{g} / \mathrm{ml}$ CMS 10-E-15-350 could not be observed (Supplementary information Figure 2 A, B).

\section{CMS 10-E-15-350 did not affect the secretion of pro- inflammatory cytokines}

Gene expression analyses revealed that the mRNAs of the pro-inflammatory cytokines IL-1 beta and TNF alpha were not altered upon nanocarrier exposure regardless of the concentration and the application time (Fig. 4c). The mRNA of IL- 6 was upregulated by the nanocarrier at the highest concentration $(500 \mu \mathrm{g} / \mathrm{ml})$ applied after 3, 6, and $12 \mathrm{~h}$ compared to corresponding control cells (Fig. 4d). For IL-8, the nanocarrier caused an upregulation of mRNA expression at concentrations of $50 \mu \mathrm{g} / \mathrm{ml}$ after $3 \mathrm{~h}$ and of $500 \mu \mathrm{g} / \mathrm{ml}$ after $3 \mathrm{~h}$ and $6 \mathrm{~h}$ (Fig. $4 \mathrm{c}$ ). However, analysis of the medium of cells treated with 50 and $500 \mu \mathrm{g} / \mathrm{ml}$ CMS 10-E-15-350 revealed that the concentration of IL- 6 and IL- 8 was not altered compared control cells (Fig. 4d). 
a

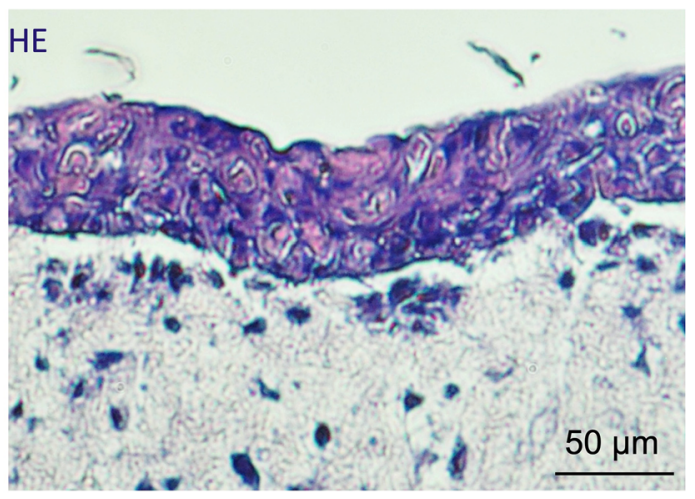

C

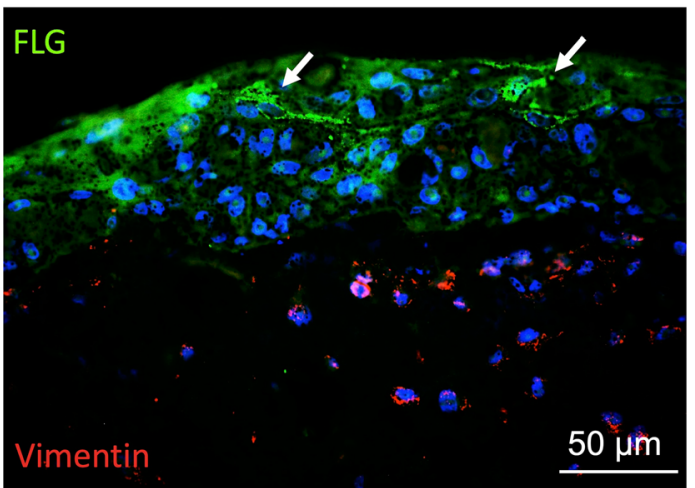

Fig. 3 Representative images of nanocarrier penetration into in vitro 3D mucosal equivalents of co-cultured gingival keratinocytes and collagen/ fibroblast gel. a Microsections were stained for hematoxylin-eosin. b Pan-cytokeratin (green) and vimentin (red). c Filaggrin and vimentin.

\section{Transepithelial electrical resistance of OKG4 cells remained unchanged in the presence of CMS nanocarriers}

To study the penetration route of the nanocarrier, we characterized the physical barrier formed by differentiating OKG4 cells (Fig. 5a-e). For this purpose, cells were grown to confluence in the presence of $60 \mu \mathrm{M} \mathrm{Ca}^{2+}$. Then, cell differentiation was induced by increasing the $\mathrm{Ca}^{2+}$ concentration in the medium to $1.4 \mathrm{mM}$ (high $\mathrm{Ca}^{2+}$ ). Under these conditions, a constantly increasing TEER was monitored with a peak at day 8 . Then, the resistance decreased but remained stable between day 12 and 24 (Fig. 5a). Cells cultivated in the presence of $60 \mu \mathrm{M}$ did not exhibit a measurable resistance (Fig. 5a).

The increase in TEER was accompanied by formation of tight cell-cell contacts (Supplementary information Figure 3 A). Cells cultured in the presence of $60 \mu \mathrm{M} \mathrm{Ca}^{2+}$ (low $\mathrm{Ca}^{2+}$ ) showed no cell-to-cell contacts (Supplementary information Figure 3 B).

Administration of CMS 10-E-15-350 affected neither the TEER of the cell monolayer compared to control cells (Fig. 5 b) nor the integrity of cell-cell contacts (Fig. 5 c, d, Supplementary information Figure 4). Using fluorescence analysis, we showed that the fluorescence-coupled b
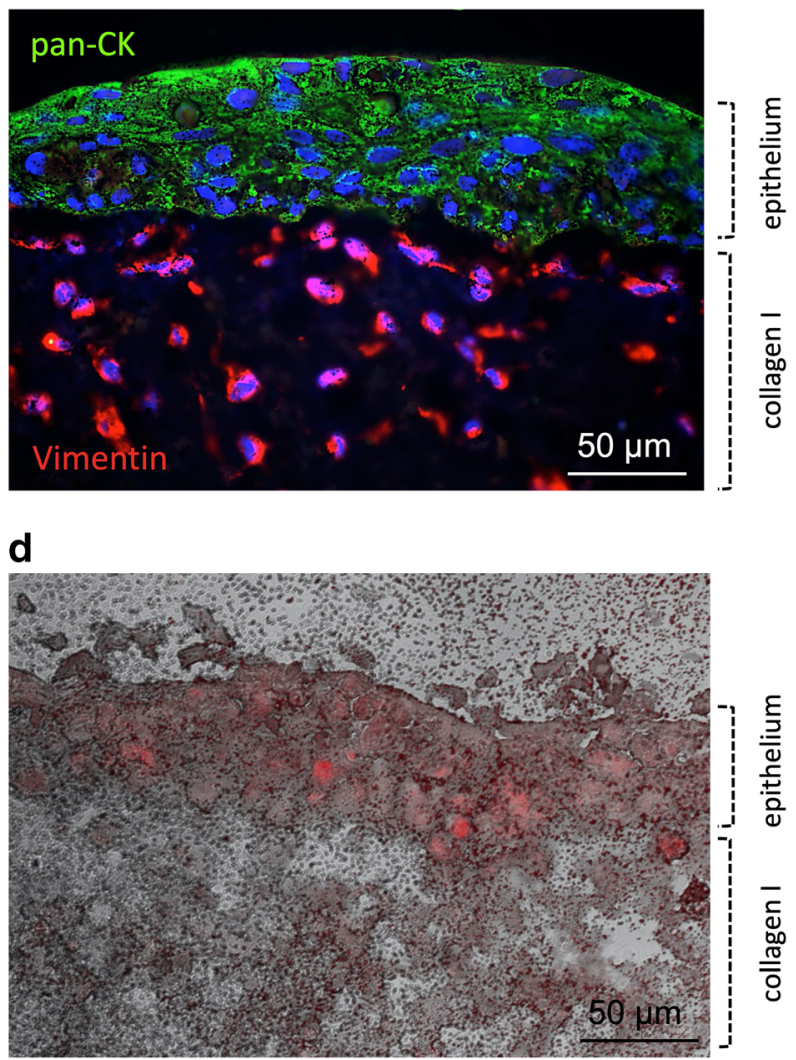

Cell nuclei were visualized with the Hoechst 33342 dye (blue). d Microscopic image of the penetrated nanocarrier coupled to the fluorescent dye indocarbocyanine (red). Magnification $\times 40$

nanocarrier (red) was taken up by differentiated (Fig. 5c) and undifferentiated cells (Fig. 5d). The counterstaining of cell nuclei with Hoechst 33342 (blue) revealed that the nanocarrier is localized perinuclearily (Fig. $5 \mathrm{c}$, d) as confirmed by orthogonal (xz) projections (Fig. 5e). In primary epithelial cells, low amounts of the CMS 10-E-15-350 were found even after $5 \mathrm{~min}$ in the cytosol. The amount of nanocarrier within the cells increased over time (Fig. 5f).

\section{Discussion}

Limited bioavailability upon topical drug application still represents the major challenge for the treatment of oral inflammatory diseases. Here, we tested the hypothesis whether a new generation of an ester-based CMS nanocarrier, CMS 10 -E-15-350, is a suitable nontoxic drug-delivery system that penetrates efficiently to oral mucosal tissues without influencing the inflammatory response, the metabolic activity, and the proliferation rate of gingival epithelial cells.

While the CMS nanocarrier penetrated relatively evenly into the para-keratinized lining mucosa, it was prone to aggregation in the stratum corneum of the masticatory mucosa. The 

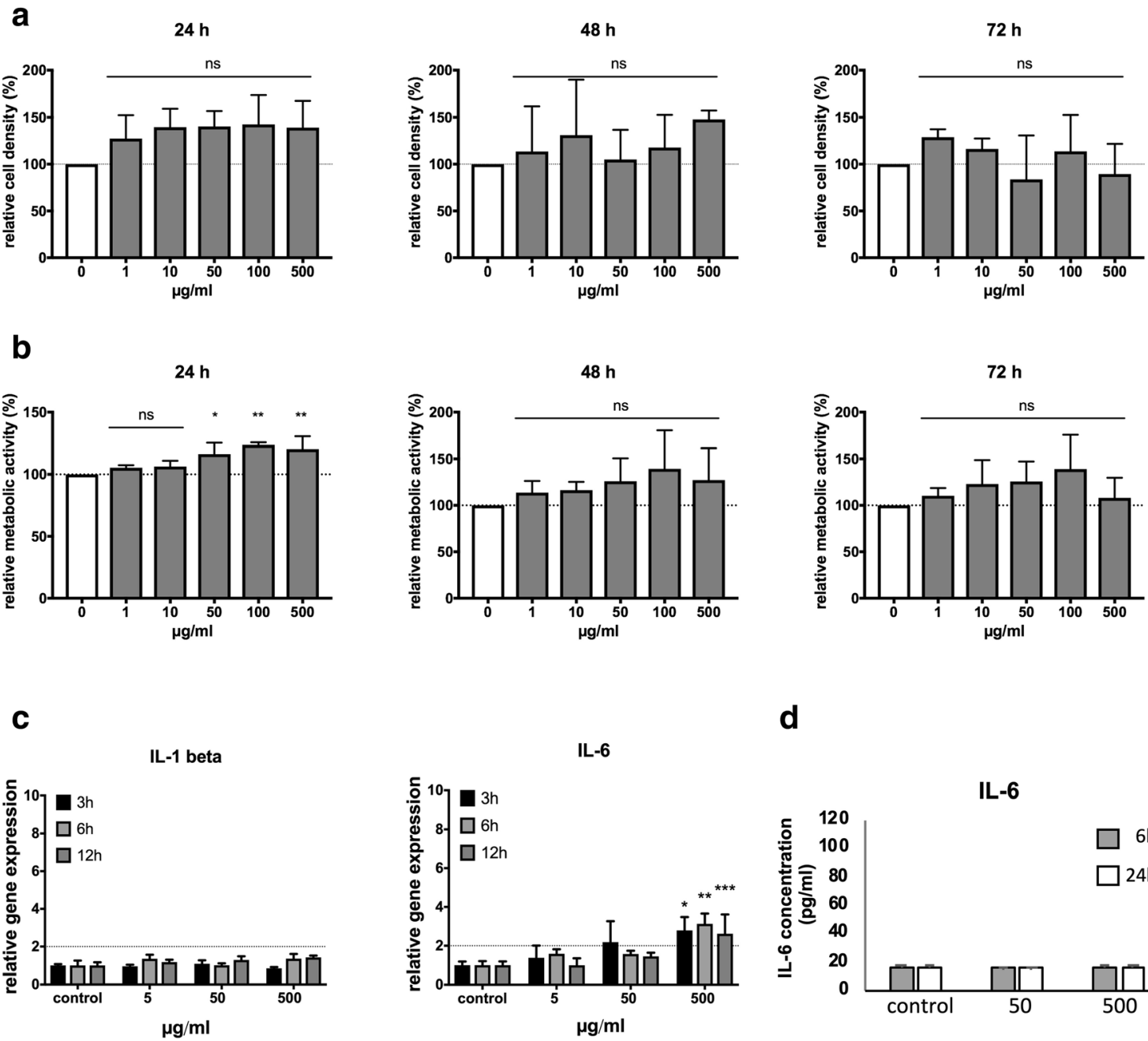

IL-6
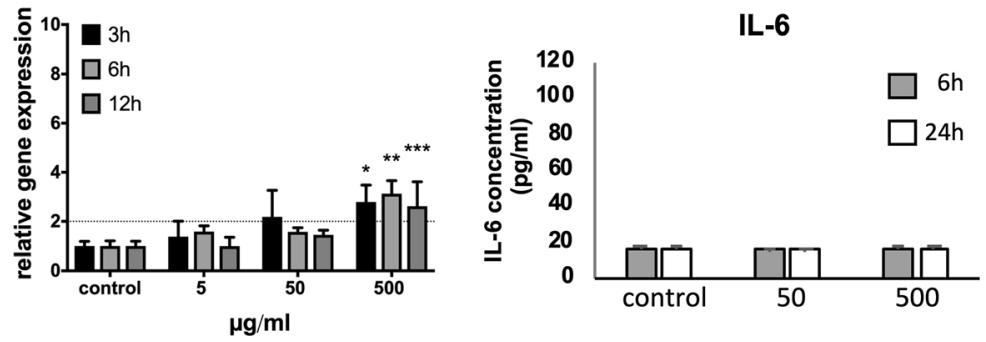

IL-8
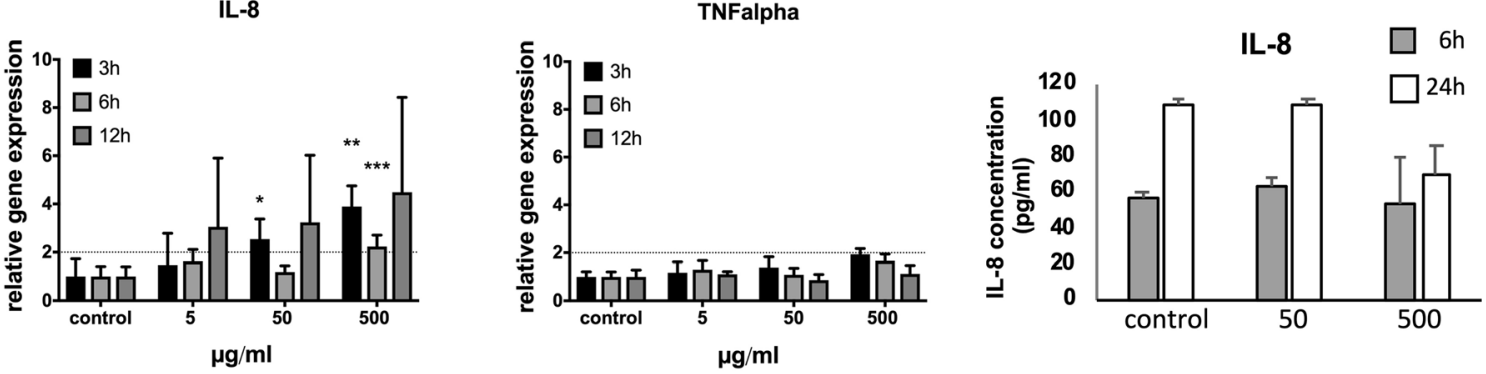

Fig. 4 a Cell proliferation assay (sulphorhodamine B). OKG4 cells were exposed to nanocarrier concentrations of $1,10,50,100$, and $500 \mu \mathrm{g} / \mathrm{ml}$ for 24, 48, and $72 \mathrm{~h}$. b Measurement of the metabolic activity (MTT) of OKG4 cell upon application of the CMS 10-E-15-350 nanocarrier under the afore mentioned conditions. $* P=0.0182$; $* * P=0.0021$. c Analysis of the mRNA expression of IL-1 beta, IL-6 ( $* P=0.0103$; ** $P=0.0001$;

*** $P=0.0001), \mathrm{IL}-8(* P=0.0416$; ** $P=0.0002 ; * * * P=0.0002)$, and TNF alpha upon nanocarrier application on gingival keratinocytes (OKG4). Gene expression analysis was performed by real-time PCR experiments. d Cytokine secretion analyses of IL-6 and IL-8 using ELISA technology

penetration behavior of the CMS 10-E-15-350 nanocarrier was in accordance with the penetration observed for the CMS 10-A-18-350 nanocarrier into the oral mucosa [23]. However, this was contrary to skin experiments which revealed that CMS nanocarriers failed to penetrate into intact skin. Here, penetration was only observed into compromised skin [31].

In contrast to our previous study, shorter nanocarrier exposure times were chosen. After $30 \mathrm{~min}$, the CMS nanocarrier penetrated into the lining mucosa to a comparable extent as 
Fig. 5 a Measurement of transepithelial electrical resistance (TEER) using OKG4 cells cultured in the presence of $\mathrm{Ca}^{2+}$ concentrations of $60 \mu \mathrm{M}$ (gray line) and $1.4 \mathrm{mM}$ (black line), respectively. b Relative TEERtures when exposed to the CMS 10-E-15-350 nanocarrier (gray trol cells (black bars) for 24, 48, and $72 \mathrm{~h}$. Visualization of nanocarrier uptake into epithelial cells (OKG4) stained with Alexa Fluor 488-coupled wheat germ agglutinin (WGA; green) and Hoechst 33342 dye (blue) in the presence of $\mathbf{c} 1.4 \mathrm{mM} \mathrm{Ca}^{2+}$ and $\mathbf{d}$ $60 \mu \mathrm{M}$. Intracellular perinuclear localization of the CMS 10-E-15350 nanocarrier coupled to the fluorescent dye indocarbocyanine (red) was monitored by CLSM (magnification $\times 63$ ), and e contion. $\mathbf{f}$ Visualization of nanocarrier uptake into primary epithelial cells over time. Cells were incubated with indocarbocyanine-coupled CMS 10-E-15-350 nanocarrier (red) for $5 \mathrm{~min}, 30 \mathrm{~min}$, and $6 \mathrm{~h}$ or left untreated. Subsequently, cells were stained with Alexa Fluor 488(WGA; green) and Hoechst 33342 dye (blue, magnification $\times 60$ ) measurements in OKG4 cell culbars) compared to untreated confirmed by an orthogonal projeccoupled wheat germ agglutinin a

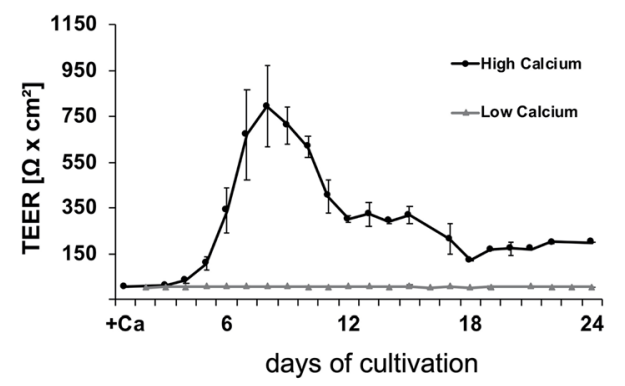

C

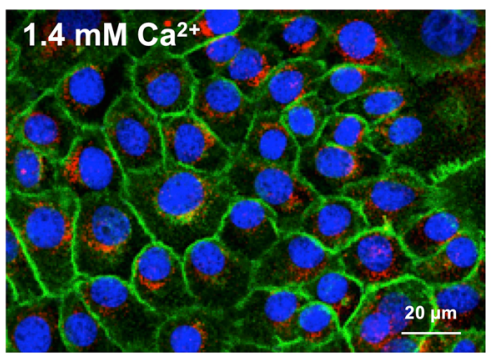

e

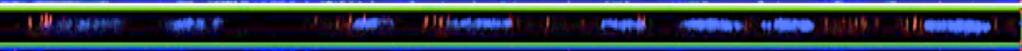

f
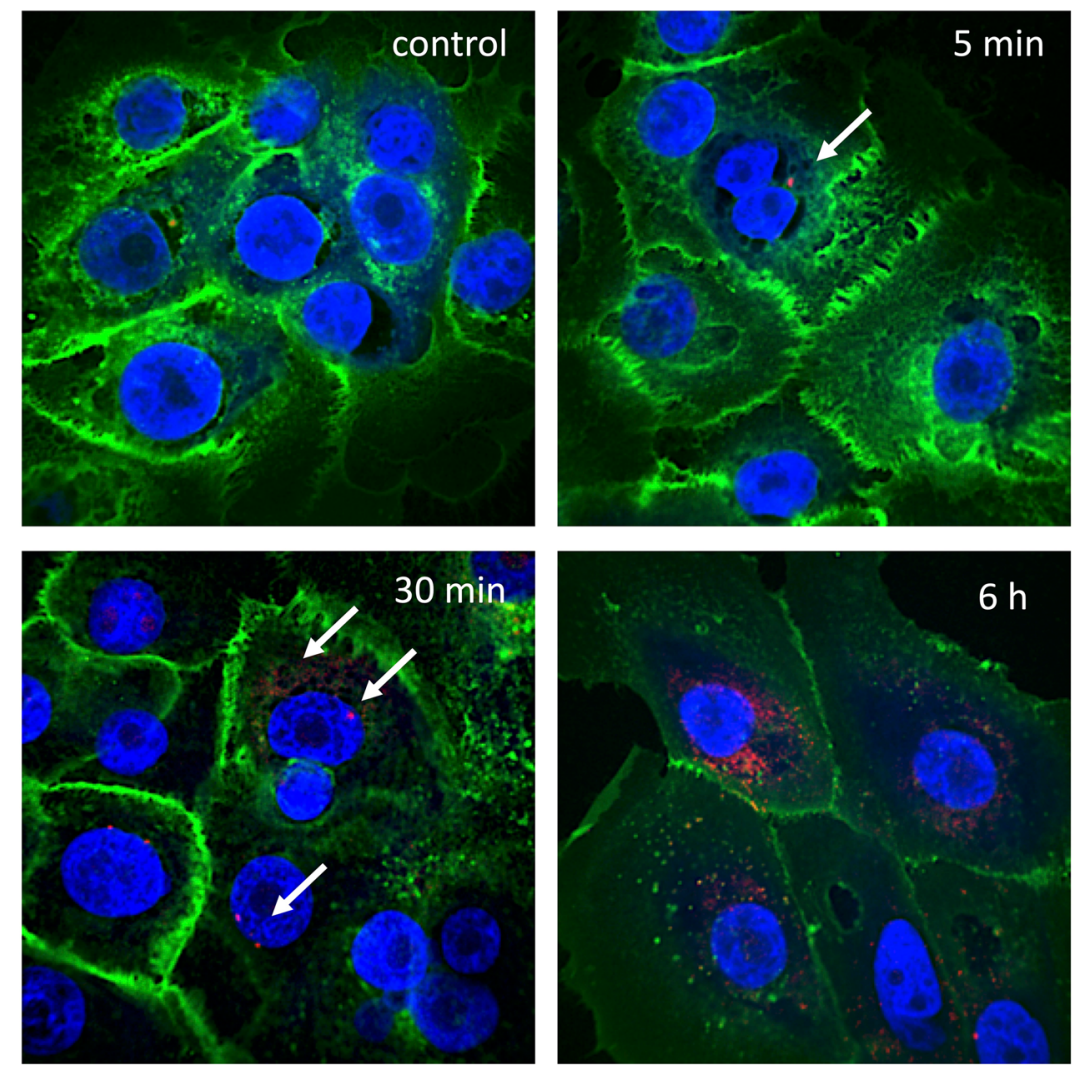

b

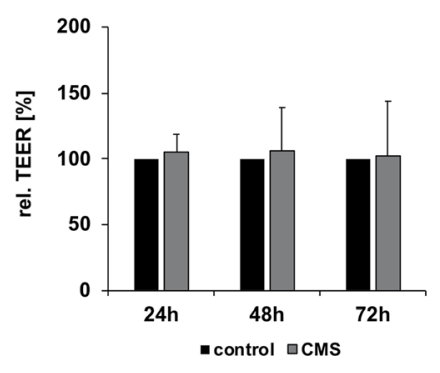

d

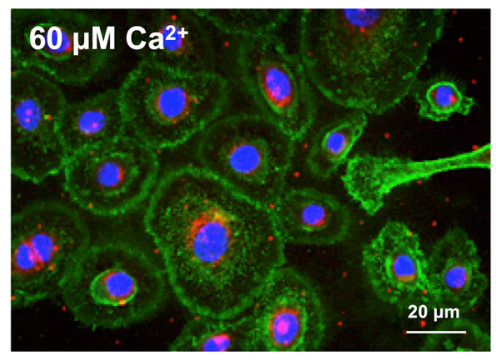

observed after 6 h [23]. Even after 5 min, nanocarrier penetration was observed but to a lesser extent compared to the longer exposure times. These findings suggest that especially in mucosal types that exhibit lower degrees of keratinization, CMS nanocarriers may reach a sufficient penetration depth even after a short application time. A penetration time within 
minutes may allow an appropriate clinical handling with a higher degree of patient's acceptance.

In general, ex vivo and/or in vivo animal experiments should be reduced or avoided whenever possible [32-34]. We have, therefore, established a novel experimental approach to monitor penetration dynamics of nanocarrier in a 3D full-thickness gingiva equivalent that is structurally comparable to human oral mucosal tissues. Similar to the ex vivo experiments, the nanocarrier penetrated through the epithelial cell layers, but not into the underlying connective tissue.

This approach will open new avenues for novel experimental designs that will facilitate research into drug delivery, release, and efficacy of anti-inflammatory compounds at the cellular and subcellular levels compared to in vivo and ex vivo experiments.

Our results showed that the nanocarrier CMS 10-E-15-350 did not interfere with the physical integrity of the mucosal tissue model. Penetration may occur by paracellular or transcellular routes. As determined by transepithelial electrical resistance (TEER) measurements, OKG4 cells developed a tight physical barrier that is comparable or superior to other similar gingiva culture models published so far [30, 35]. Nanocarrier treatment of cells had no impact on the cell monolayer's resistance. Furthermore, the ICC-coupled nanocarrier was found intracellularly after administration using confocal microscopy. These results favor a transcellular route into the oral mucosa. Similar intracellular pathways have been described reflecting processes also involved in autophagy (endosome/exosome secretory pathway) [36]. For the Langerhans cell line XS52, it has been shown that CMS nanocarrier is not taken up by one exclusive pathway, but by micropinocytosis, caveolaemediated endocytosis, and clathrin-mediated endocytosis [37]. In this study, a perinuclear localization of CMS 10-E15-350 was determined by CLSM analyses. Whether the CMS nanocarrier utilizes similar or specific cellular uptake mechanisms needs to be determined in future investigations.

Biosafety is an important issue in biomedical nanotechnology. Thus, nanoparticles should be bioinert or biodegradable and their use should not cause toxic side effects [38, 39].

The ester-based CMS 10-E-15-350 nanocarriers have been designed to produce hyperbranched polyglycerol (hPG), alkyl diacids, and mPEG350 on degradation to improve biocompatibility over amide-based CMS nanocarriers [40]. We were able to show that the CMS 10-E-15-350 nanocarrier caused no significant changes in cell proliferation or metabolic activity in gingival epithelial cells and may therefore be more suitable for the application at the oral mucosa compared to the amide-based CMS 10-A-18-350 nanocarrier which exhibited cytotoxic effects at high concentrations and after long exposure times in gingival epithelial cells [23]. The CMS 10-E-15350 nanocarrier increased the metabolic activity merely at high concentrations within the first $24 \mathrm{~h}$. This early activity may occur in conjunction with the intracellular uptake of the nanocarrier which was observed after $24 \mathrm{~h}$. These data are in line with studies performed in skin models which showed that the CMS 10-E-15-350 nanocarrier demonstrated excellent biocompatibility as assessed in comprehensive toxicological assays [25].

Furthermore, it was demonstrated that the mRNA expression of IL-6 and IL-8 was upregulated only at the highest nanocarrier concentration $(500 \mu \mathrm{g} / \mathrm{ml})$ applied. The mRNA expression of the other pro-inflammatory mediators IL-1 beta, CCL-20, and TNF alpha remained unaffected. This suggests that CMS 10-E-15-350 nanocarrier should be applied at lower concentrations.

In conclusion, the present study introduced the newly developed core-multi shell nanocarrier CMS 10-E-15-350 to oral mucosal models and cells for the first time. This nanocarrier revealed fast adherence and penetration properties into the epithelial cell layer of oral mucosal tissues. Within the limitations of this study, the results of in vitro experiments indicated a transcellular penetration pathway into the epithelial tissue, and the transepithelial resistance, which is an indicator of the integrity of the physical barrier, remained unchanged. Physiological CMS concentrations did not provoke alterations in the cellular metabolic activity, the cellular proliferative status, or the immune response. Thus, this biodegradable nanocarrier may be considered biologically safe regarding its application onto oral mucosal tissues. Since this nanocarrier possesses the capability to encapsulate immunologically relevant drugs, such as dexamethasone and eternacept [17, 25], this technology opens new avenues for promising therapeutic approaches in the context of translational science for the treatment of oral diseases.

Supplementary Information The online version contains supplementary material available at https://doi.org/10.1007/s00784-021-03884-x.

Acknowledgements The authors kindly thank Mrs. Gudrun Mrawietz, Mrs. Marion von Zitzewitz, Mrs. Verena Kanitz, and Jan Viktorov for their excellent technical assistance.

Author contribution H.D.: contributed to the conception, design, data acquisition, and interpretation, performed statistical analyses, and drafted and critically revised the manuscript.

K.S.: contributed to the conception, data acquisition, and interpretation, and drafted and critically revised the manuscript, additional experiments during the revision process.

J.J.: contributed to the design, data acquisition, and interpretation, and critically revised the manuscript.

K.V.: contributed to the design and data acquisition, and critically revised the manuscript.

R.W.: contributed to the design, data acquisition, and interpretation, and critically revised the manuscript.

S.H.: contributed to the conception and data interpretation, and critically revised the manuscript.

M.U.: contributed to the design and data interpretation, and drafted and critically revised the manuscript. 
R.H.: contributed to the conception, design, and data interpretation, and drafted and critically revised the manuscript.

K.D.: contributed to the conception, design, data acquisition, and interpretation, and drafted and critically revised the manuscript.

All authors gave their final approval and agreed to be accountable for all aspects of the work.

Funding Open Access funding enabled and organized by Projekt DEAL. This study was supported by the German Research Foundation (Deutsche Forschungsgemeinschaft, DFG: H.D.: DO 1375/2-1; K.D.: DA 310/8-1; R.H.: HA 2549/22-1). KN.S. and J.J. were financially supported by the Sonnenfeld-Stiftung.

\section{Declarations}

All experiments followed the guidelines of good clinical/laboratory practice (GCP/GLP) and the WHO Declaration of Helsinki 1964, latest update Seoul 2008 (59th WMA General Assembly, Seoul, October 2008). The study was approved by the ethical committee of the Charite Universitätsmedizin Berlin (IRB number: EA2/185/16). All volunteers gave their informed consent.

Conflict of interest The authors declare no competing interests.

Open Access This article is licensed under a Creative Commons Attribution 4.0 International License, which permits use, sharing, adaptation, distribution and reproduction in any medium or format, as long as you give appropriate credit to the original author(s) and the source, provide a link to the Creative Commons licence, and indicate if changes were made. The images or other third party material in this article are included in the article's Creative Commons licence, unless indicated otherwise in a credit line to the material. If material is not included in the article's Creative Commons licence and your intended use is not permitted by statutory regulation or exceeds the permitted use, you will need to obtain permission directly from the copyright holder. To view a copy of this licence, visit http://creativecommons.org/licenses/by/4.0/.

\section{References}

1. Meyle J (2000) Chapple I (2015) Molecular aspects of the pathogenesis of periodontitis. Periodontology 69(1):7-17. https://doi.org/ 10.1111/prd.12104

2. Page RC (2000) Kornman KS (1997) The pathogenesis of human periodontitis: an introduction. Periodontology 14:9-11

3. Kassebaum NJ, Bernabe E, Dahiya M, Bhandari B, Murray CJ, Marcenes W (2014) Global burden of severe periodontitis in 1990-2010: a systematic review and meta-regression. J Dent Res 93(11):1045-1053. https://doi.org/10.1177/0022034514552491

4. Keestra JA, Grosjean I, Coucke W, Quirynen M, Teughels W (2015) Non-surgical periodontal therapy with systemic antibiotics in patients with untreated aggressive periodontitis: a systematic review and meta-analysis. J Periodontal Res 50(6):689-706. https://doi.org/10.1111/jre.12252

5. Keestra JA, Grosjean I, Coucke W, Quirynen M, Teughels W (2015) Non-surgical periodontal therapy with systemic antibiotics in patients with untreated chronic periodontitis: a systematic review and meta-analysis. J Periodontal Res 50(3):294-314. https://doi. org/10.1111/jre.12221

6. Harks I, Koch R, Eickholz P, Hoffmann T, Kim TS, Kocher T, Meyle J, Kaner D, Schlagenhauf U, Doering S, Holtfreter B, Gravemeier M, Harmsen D, Ehmke B (2015) Is progression of periodontitis relevantly influenced by systemic antibiotics? A clinical randomized trial. J Clin Periodontol 42(9):832-842. https://doi.org/10.1111/jcpe.12441

7. van Winkelhoff AJ, Herrera D, Oteo A, Sanz M (2005) Antimicrobial profiles of periodontal pathogens isolated from periodontitis patients in The Netherlands and Spain. J Clin Periodontol 32(8):893-898. https://doi.org/10.1111/j.1600-051X.2005.00782.

8. van Winkelhoff AJ, Herrera Gonzales D, Winkel EG, DellemijnKippuw N, Vandenbroucke-Grauls CM, Sanz M (2000) Antimicrobial resistance in the subgingival microflora in patients with adult periodontitis. A comparison between The Netherlands and Spain. J Clin Periodontol 27(2):79-86

9. Van Dyke TE, Hasturk H, Kantarci A, Freire MO, Nguyen D, Dalli J, Serhan CN (2015) Proresolving nanomedicines activate bone regeneration in periodontitis. J Dent Res 94(1):148-156. https:// doi.org/10.1177/0022034514557331

10. Hasturk H, Kantarci A, Van Dyke TE (2012) Paradigm shift in the pharmacological management of periodontal diseases. Front Oral Biol 15:160-176. https://doi.org/10.1159/000329678

11. Schifter M, Yeoh SC, Coleman H, Georgiou A (2010) Oral mucosal diseases: the inflammatory dermatoses. Aust Dent J 55(Suppl 1): 23-38. https://doi.org/10.1111/j.1834-7819.2010.01196.x

12. Atzori L, Pau M, Podda R, Manieli C, Aste N (2011) A case of bullous pemphigoid in infancy treated with local corticosteroids. G Ital Dermatol Venereol 146(6):493-496

13. Zadik Y, Elad S, Shapira A, Shapira MY (2017) Treatment of oral mucosal manifestations of chronic graft-versus-host disease: dexamethasone vs. budesonide. Expert Opin Pharmacother 18(3):235242. https://doi.org/10.1080/14656566.2017.1282464

14. Proctor GB (2016) The physiology of salivary secretion. Periodontol 70(1):11-25. https://doi.org/10.1111/prd.12116

15. Taylor JJ (2000) Preshaw PM (2016) Gingival crevicular fluid and saliva. Periodontology 70(1):7-10. https://doi.org/10.1111/prd. 12118

16. Donos N (2018) The periodontal pocket. Periodontol 76(1):7-15. https://doi.org/10.1111/prd.12203

17. Giulbudagian M, Yealland G, Honzke S, Edlich A, Geisendorfer B, Kleuser B, Hedtrich S, Calderon M (2018) Breaking the barrierpotent anti-inflammatory activity following efficient topical delivery of etanercept using thermoresponsive nanogels. Theranostics 8(2):450-463. https://doi.org/10.7150/thno.21668

18. Zhou Q, You C, Ling Y, Wu H, Sun B (2019) pH and thermo dual stimulus-responsive liposome nanoparticles for targeted delivery of platinum-acridine hybrid agent. Life Sci 217:41-48. https://doi.org/ 10.1016/j.lfs.2018.11.052

19. Bruun J, Larsen TB, Jolck RI, Eliasen R, Holm R, Gjetting T, Andresen TL (2015) Investigation of enzyme-sensitive lipid nanoparticles for delivery of siRNA to blood-brain barrier and glioma cells. Int J Nanomedicine 10:5995-6008. https://doi.org/10.2147/ IJN.S87334

20. Xu C, Song RJ, Lu P, Chen JC, Zhou YQ, Shen G, Jiang MJ, Zhang $\mathrm{W}$ (2018) $\mathrm{pH}$-triggered charge-reversal and redox-sensitive drugrelease polymer micelles codeliver doxorubicin and triptolide for prostate tumor therapy. Int J Nanomedicine 13:7229-7249. https:// doi.org/10.2147/IJN.S182197

21. Burakowska E, Zimmerman SC, Haag R (2009) Photoresponsive crosslinked hyperbranched polyglycerols as smart nanocarriers for guest binding and controlled release. Small 5(19):2199-2204. https://doi.org/10.1002/smll.200900465

22. Mura S, Nicolas J, Couvreur P (2013) Stimuli-responsive nanocarriers for drug delivery. Nat Mater 12(11):991-1003. https://doi.org/10.1038/nmat3776

23. Jager J, Obst K, Lohan SB, Viktorov J, Staufenbiel S, Renz H, Unbehauen M, Haag R, Hedtrich S, Teutloff C, Meinke MC, Danker K, Dommisch H (2018) Characterization of hyperbranched core-multishell nanocarriers as an innovative drug delivery system 
for the application at the oral mucosa. J Periodontal Res 53(1):5765. https://doi.org/10.1111/jre.12487

24. Unbehauen M, Fleige E, Paulus F, Schemmer B, Mecking S, Moré SD, Haag R (2017) Biodegradable core-multishell nanocarriers: influence of inner shell structure on the encapsulation behavior of dexamethasone and tacrolimus. Polymers 9(8):316-332

25. Honzke S, Gerecke C, Elpelt A, Zhang N, Unbehauen M, Kral V, Fleige E, Paulus F, Haag R, Schafer-Korting M, Kleuser B, Hedtrich S (2016) Tailored dendritic core-multishell nanocarriers for efficient dermal drug delivery: a systematic top-down approach from synthesis to preclinical testing. J Control Release 242:50-63. https://doi.org/10.1016/j.jconrel.2016.06.030

26. Lindberg K, Rheinwald JG (1990) Three distinct keratinocyte subtypes identified in human oral epithelium by their patterns of keratin expression in culture and in xenografts. Differentiation 45(3):230 241

27. Dickson MA, Hahn WC, Ino Y, Ronfard V, Wu JY, Weinberg RA, Louis DN, Li FP, Rheinwald JG (2000) Human keratinocytes that express hTERT and also bypass a p16(INK4a)-enforced mechanism that limits life span become immortal yet retain normal growth and differentiation characteristics. Mol Cell Biol 20(4):1436-1447

28. Dongari-Bagtzoglou A, Kashleva H (2006) Development of a highly reproducible three-dimensional organotypic model of the oral mucosa. Nat Protoc 1(4):2012-2018. https://doi.org/10.1038/ nprot.2006.323

29. Presland RB, Dale BA (2000) Epithelial structural proteins of the skin and oral cavity: function in health and disease. Crit Rev Oral Biol Med 11(4):383-408

30. Meyle J, Gultig K, Rascher G, Wolburg H (1999) Transepithelial electrical resistance and tight junctions of human gingival keratinocytes. J Periodontal Res 34(4):214-222

31. Alnasif N, Zoschke C, Fleige E, Brodwolf R, Boreham A, Ruhl E, Eckl KM, Merk HF, Hennies HC, Alexiev U, Haag R, Kuchler S, Schafer-Korting M (2014) Penetration of normal, damaged and diseased skin-an in vitro study on dendritic core-multishell nanotransporters. J Control Release 185:45-50. https://doi.org/10. 1016/j.jconrel.2014.04.006
32. Sauer UG (2009) Animal and non-animal experiments in nanotechnology-the results of a critical literature survey. ALTEX 26(2):109-128

33. Laman JD, Kooistra SM, Clausen BE (2017) Reproducibility issues: avoiding pitfalls in animal inflammation models. Methods Mol Biol 1559:1-17. https://doi.org/10.1007/978-1-4939-6786-5_1

34. Rai J, Kaushik K (2018) Reduction of animal sacrifice in biomedical science $\&$ research through alternative design of animal experiments. Saudi Pharm J 26(6):896-902. https://doi.org/10.1016/j. jsps.2018.03.006

35. Teubl BJ, Absenger M, Frohlich E, Leitinger G, Zimmer A, Roblegg E (2013) The oral cavity as a biological barrier system: design of an advanced buccal in vitro permeability model. Eur J Pharm Biopharm 84(2):386-393. https://doi.org/10.1016/j.ejpb. 2012.10.021

36. Papandreou ME, Tavernarakis N (2017) Autophagy and the endo/ exosomal pathways in health and disease. Biotechnol J 12(1). https://doi.org/10.1002/biot.201600175

37. Edlich A, Volz P, Brodwolf R, Unbehauen M, Mundhenk L, Gruber AD, Hedtrich S, Haag R, Alexiev U, Kleuser B (2018) Crosstalk between core-multishell nanocarriers for cutaneous drug delivery and antigen-presenting cells of the skin. Biomaterials 162: 60-70. https://doi.org/10.1016/j.biomaterials.2018.01.058

38. Virlan MJ, Miricescu D, Radulescu R, Sabliov CM, Totan A, Calenic B, Greabu M (2016) Organic nanomaterials and their applications in the treatment of oral diseases. Molecules 21(2). https:// doi.org/10.3390/molecules 21020207

39. Ciappellano SG, Tedesco E, Venturini M, Benetti F (2016) In vitro toxicity assessment of oral nanocarriers. Adv Drug Deliv Rev 106: 381-401. https://doi.org/10.1016/j.addr.2016.08.007

40. Khandare J, Mohr A, Calderon M, Welker P, Licha K, Haag R (2010) Structure-biocompatibility relationship of dendritic polyglycerol derivatives. Biomaterials 31(15):4268-4277. https:// doi.org/10.1016/j.biomaterials.2010.02.001

Publisher's note Springer Nature remains neutral with regard to jurisdictional claims in published maps and institutional affiliations. 